\title{
A micromechanical modelling approach for predicting particle dislodgement
}

\author{
WURONG SHIH \& PANAYIOTIS DIPLAS
}

Imbt Environmental Hydraulics Laboratory, Department of Civil \& Environmental Engineering, Lehigh University, USA wr.shih@lehigh.edu

\begin{abstract}
In recent years, the impulse and energy concepts have been proposed as a means of improving the prediction of sediment particle entrainment. Because they account for temporal aspects of the applied hydrodynamic forces, impulse-based or energy-based approaches provide a better representation of a highly fluctuating phenomenon compared to the more traditional time-averaged Shields parameter or constant force magnitude-based model. In the present paper, we elaborate further on the simulation of particle migration by applying a theoretical model based on hydrodynamic pressure data. Three common movement scenarios and the dynamic critical drag force, allowed to vary during particle motion, are qualitatively discussed here. Also, the possible inaccuracy in entrainment prediction of three different models is addressed with reference to our simulation results. It is demonstrated that the multi-parcel impulse model has the best performance when compared to the single-parcel impulse and force magnitude-based models.
\end{abstract}

Key words impulse; hydrodynamic forces; particle dislodgement; multi-parcel approach; dynamic resistance

\section{INTRODUCTION}

Sediment dynamics in rivers has been a central topic in geomorphology for a very long time. Yet, because of the complex nature of turbulent flow and the intricate interaction between sediment particles and ambient fluid, it remains poorly understood. Among different research topics, a major effort has been devoted to the phenomenon of incipient motion of sediment particles. During the past few years, new approaches such as impulse, work, and energy have been proposed to overcome the limitations of the formulation based on the time-averaged Shields parameter and thus improve the prediction of the initiation of sediment particle movement (Diplas et al., 2008; Lee et al., 2012; Valyrakis et al., 2013). The main purpose of these efforts is to relate the fluctuating nature of turbulent flows with the "energy barrier" (Lee et al., 2012) that a particle needs to overcome and thus escape from its resting pocket. Such an escape is commonly referred to as the "migration" or "dislodgement" of the particle.

In an impulse-based model, time integration of applied hydrodynamic forces, i.e. impulse, is the fundamental quantity considered for evaluating the probability of particle dislodgement. The bounds (or limits) of integration are the boundaries of excessive forces above a threshold, or the minimum force required to move the particles. Accordingly, temporal flow data like a force trace or a velocity trace would be grouped into separate, alternatively appearing positive and negative parcels (Fig. 1). A positive force parcel, for instance, contains successive hydrodynamic forces whose magnitude suffices to initiate particle motion. The impulse contained within each parcel, then, is used to determine its individual ability to dislodge a sediment particle by comparing it with the required impulse for dislodgement. In other words, the impulse model employed here is a discrete, single-parcel approach.

However, given the continuous interplay between sediment particles and turbulence fluctuations, the single-parcel impulse approach may not account for all possible scenarios of particle movement behaviour. As a result, the critical condition of dislodgement is not a clear-cut boundary but instead a narrow band of impulse within which both movement and non-movement could occur. That leaves uncertainties in the prediction of particle migration (Celik et al., 2010, 2013). A more refined and general approach, accounting for a sequence of consecutive fluid parcels, is considered here in an effort to describe a wider range of possible particle movement scenarios.

To properly depict the progress of particle dislodgement, this paper employs a theoretical model to simulate the response of a particle to hydrodynamic forces. In this model, a perfect rolling mode is assumed for particle movement, and the applied forces upon the particle are 


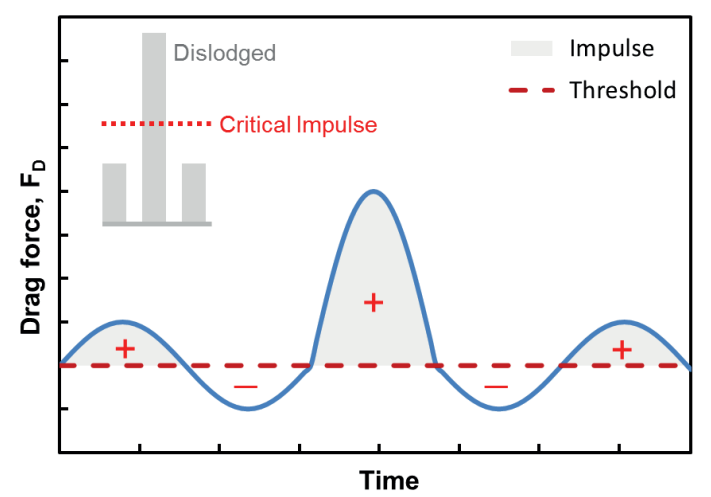

Fig. 1 Force parcels in impulse-based model.

computed from hydrodynamic pressure data measured by Celik et al. (2013). In addition to the migration trajectory, the corresponding variation in particle resistance is shown here. Above all, we intend to provide evidence that supports the advantage of a multi-parcel approach over the single-parcel approach in terms of predicting particle dislodgement.

In the following sections, the experimental arrangement, various movement scenarios, dynamic particle resistance and the mechanical details are presented. Finally, we discuss the possible inaccuracy of several approaches to predict dislodgement.

\section{EXPERIMENT AND EQUATIONS OF MOTION}

To determine the hydrodynamic forces applied on a sediment particle, the results from a laboratory study reported in Celik et al. (2013) are employed in this paper. In their experiments, four synchronized pressure transducers are mounted on a target sphere of $12.7 \mathrm{~mm}$ in diameter, which is fixed within a pocket formed by three hexagonal, well-packed identical spheres (Fig. 2(a)). Pressure transducers P1 and P2 are set along the centreline on the front and back sides of the sphere to estimate the drag forces. Transducers P3 and P4 are placed on the top and bottom of this spherical grain, respectively, for determining the lift forces (Fig. 2(b)).

The drag and lift force traces are determined from the corresponding pressure data recorded for 15 minutes. For simplicity, the resultant drag, $F_{D}$, and lift, $F_{L}$, forces are estimated by:

$$
\begin{aligned}
& F_{D}=\left(p_{1}-p_{2}\right) A_{P} \\
& F_{L}=\left(p_{4}-p_{3}\right) A_{P}
\end{aligned}
$$

Here the lower case " $p$ " represents corresponding pressure data from the individual pressure transducers. The area of the particle projected in a direction perpendicular to the flow is $A_{P}=$ $e(\pi / 4) d^{2}$, and the exposure factor is $e=0.85$ for drag force calculation and $e=1.0$ for lift force computation. Once the flow forces are available, their resultant torque, $T$, is determined according to the in situ geometry:

$$
\begin{aligned}
& T=\left(F_{D}+f_{v} W_{s} \sin \beta\right) Z-\left(f_{v} W_{S} \cos \beta-F_{L}\right) X \\
& X=(d / 2) \sin (\pi / 3) \sin (\theta+\beta) \\
& Z=(d / 2) \sin (\pi / 3) \cos (\theta+\beta)
\end{aligned}
$$

Here, the hydrodynamic mass coefficient is $f_{v}=1+0.5\left(\rho_{f} /\left(\rho_{s}-\rho_{f}\right)\right)$, and the sphere weight is $W_{S}=$ $\rho_{s}(\pi / 6) d^{3}$. The density of a Teflon sphere is used in simulation with $\rho_{s}=2300 \mathrm{~kg} / \mathrm{m}^{3}$, and the density of water is $\rho_{f}=1000 \mathrm{~kg} / \mathrm{m}^{3} . X=$ lever arm for vertical forces and $Z=$ lever arm for longitudinal forces (Fig. 2(c)). The resistance angle, $\theta$, changes with sphere trajectory, whereas the bed slope, $\beta=0.25 \%$, remains constant during the experiments. 


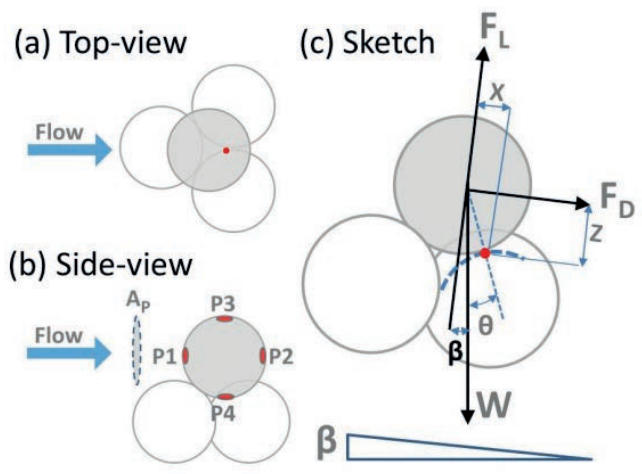

Fig. 2 Sketch of experimental arrangement.

A theoretical simulation of particle movement, hence, is applied based to the so obtained torque values. Starting from the onset of a positive force parcel, the in situ torque and the angulardisplacement deriving from this torque are computed progressively with a time interval of 0.004 seconds. Due to the perfect-rolling assumption, the particle location could be updated continuously with the latest angular-displacement. The effect of particle dislocation on the applied hydrodynamic forces is neglected because of our limited knowledge of the corresponding turbulent flow. Note that the simulation is terminated at the full dislodgement moment instead of the end of the positive force parcel. In other words, a full dislodgement detected here may span more than one parcel; thus, the present model belongs to the multi-parcel approach.

A sediment particle is seen as fully dislodged once it overcomes the angle of repose, $\varnothing$, and reaches the apex of base spheres where no particle resistance to rolling would exist. With the compact bed formation of three identical spheres, the angle of repose is given by Julien (2010):

$$
\tan \phi=\frac{1}{\sqrt{3\left(1+\frac{d_{1}}{d_{2}}\right)^{2}-4}}
$$

Here, $d_{1}$ is the diameter of three base spheres and $d_{2}$ the diameter of the top sphere. In the present case all four spheres are identical in size, $d=12.7 \mathrm{~mm}$, and the computed angle of repose is $\varnothing=$ $19.5^{\circ}=.34(\mathrm{rad})$. The completeness of dislodgement, therefore, is the ratio of cumulative angulardisplacement to this obtained angle of repose.

The simulation is started over upon one full dislodgement and the same procedure is repeated to detect all particle movement during the 15-min data. The migration trajectory, in situ particle resistance, and corresponding mechanical aspects are now available for further discussion.

\section{MOVEMENT SCENARIOS}

Several movement scenarios are found in the simulation, including incomplete dislodgements, prompt dislodgements, and tardy dislodgements. All failed attempts of migration are referred to as the incomplete dislodgements. These incomplete events usually result in the vibrating phenomena as the particle falls back to the resting spot with momentum (Fig. 3(a)). For those being dislodged, the term "prompt" depicts the monotonically advancing particle that achieves dislodgement instantly (Fig. 3(b)); whereas, the term "tardy" represents the particle migration accompanied by reverse movement, which takes longer duration to complete (Fig. 3(c)). Some dislodgements are discovered to closely follow the vibration resulting from the previous incomplete movement. Those successful migrations, most of the time, can be seen as the extension of their unsuccessful forerunners and hence are referred to as vibrational dislodgements (Fig. 3(d)).

Figure 3(b)-(d) depict the progress of the prompt, tardy and vibrational migrations, respectively. As mentioned, the completeness of dislodgement on the y-axis is the ratio of cumulative angular-displacement to the angle of repose. The value of unity represents the fully- 
(a) Incomplete dislodgement

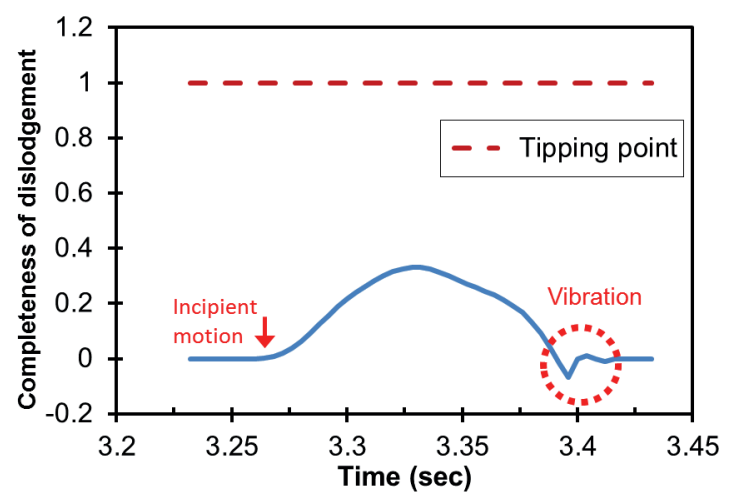

(c) Tardy dislodgement

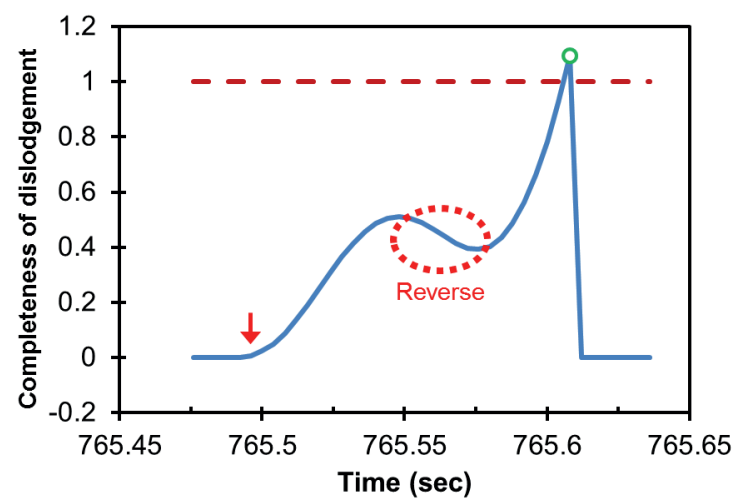

Fig. 3 Types of particle dislodgement. (b) Prompt dislodgement

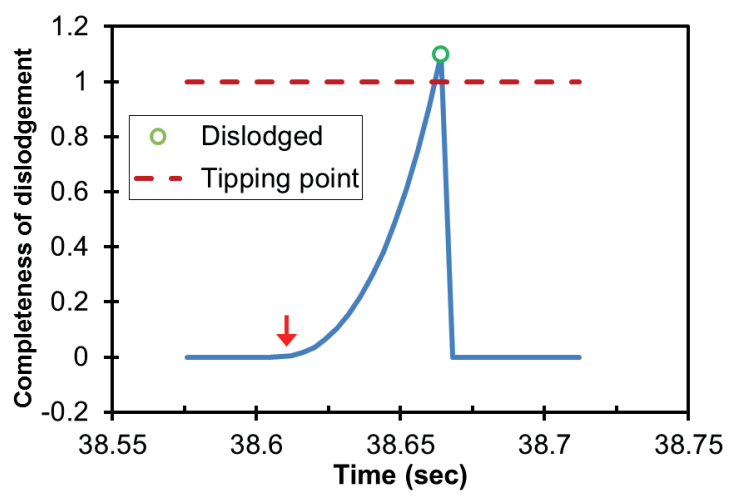

(d) Vibrational tardy dislodgement

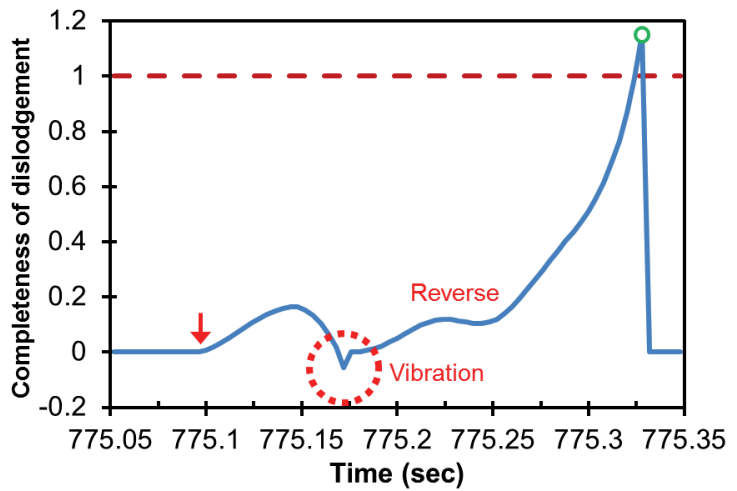

dislodged status of the sediment particle. Starting at zero, the motion toward downstream increases the completeness, whereas the motion backward upstream reduces its value. The particle trajectory, therefore, is presented by the trace of migration completeness in Fig. 3(b)-(d).

In a case of being promptly dislodged (Fig. 3(b)), the particle moves downstream without experiencing any apparent resistance. The completeness increases monotonically and reaches the tipping point within 0.1 seconds. This entrainment duration is of the same order as the value observed by $\mathrm{Wu} \&$ Shih (2012). Based on the increasing slope of the completeness trace, the particle is found to retain acceleration over this migration; that implies the hydrodynamic forces always exceed the particle resistance during this period (positive external forces). This implication is verified by the corresponding mechanical aspects shown in the following sections.

Figure 3(c) shows a reverse motion stage existing in the tardy dislodgement. As marked by a red-dashed circle, the dented section represents the particle falling back toward the resting pocket. The reverse movement, however, is supressed by flow before the particle arrives at the pocket bottom. Then, the particle resumes moving downstream and achieves dislodgement afterwards. The reverse in particle trajectory reveals the inability of certain flow forces to support the particle. More important, the two-stage acceleration suggests that two separate positive force parcels are involved in this migration. That emphasizes the necessity of a multi-parcel approach.

As shown in Fig. 3(d), a tardy dislodgement is found to follow the vibration of an incomplete migration. The vibrating stage appears in negative angular-displacement as the particle rotates upstream and leans upon the upstream base-particle instead of the downstream one. This backward movement persists for only about 0.01 seconds before being suppressed by the coming flow and gravity. Upon the vibration, the subsequent energetic flow results in a complete migration, though slightly reverse movement is noticed here. Given the complex trajectory of vibration-accelerationreverse-acceleration, at least three positive force parcels are anticipated during this movement. 
The present simulation shows a wide variety of particle dislodgements: some of them appear in a monotonically advancing trajectory, whereas others show a complex migrating progress that involves variant particle dynamics. The later migration type indicates the continual interplay between sediment particles and turbulent fluctuations. This incessant influence of flow, however, cannot be properly represented by the single-parcel approach, since it focuses on individual force parcels and thus neglects the combined effect of successive parcels.

In addition to fluctuating flow forces, the varying particle resistance complicates the particle dynamics. The following section elaborates on this dynamic resistance and its effect as well.

\section{DYNAMIC PARTICLE RESISTANCE}

Particle resistance, or the critical drag force to commence particle motion $\left(F_{D c r}\right)$, is allowed to vary throughout the present simulation. To determine this threshold, various parameters are included such as particle weight, bed geometry, and hydrodynamic lift forces. Accordingly, the equation based on the balance of moment is given by:

$$
F_{D c r}=\left(f_{v} W_{S} \cos \beta-F_{L}\right) \tan (\theta+\beta)-f_{v} W_{S} \sin \beta
$$

Provided particle weight, $W_{S}$, and bed slope, $\beta$, are constant here, the threshold merely depends on the resistance angle, $\theta$, and fluctuating lift forces, $F_{L}$. The resistance angle changes with particle trajectory that is determined by the resultant torque, which involves lift forces. An intricate nonlinear relationship, therefore, is found among these three parameters. For simplicity, this paper qualitatively elaborates on the effects of the resistance angle and lift forces upon the threshold, respectively.

Figure 4(a) shows the dynamic threshold of three particle densities versus the resistance angle non-dimensionalized by the angle of repose. No lift forces are considered here. As expected, a sediment particle with higher density requires a larger drag force to initiate its movement. With the reduction in the resistance angle, i.e. increment in migration completeness, the threshold decreases linearly until the tipping point, where no particle resistance exists. Given the reducing threshold over time, an overestimate of the impulse request for migration is found when the initial critical value is taken as the universal threshold. In other words, the probability of dislodgement is underrated as a constant threshold is applied.

As to the effect of lift forces, Fig. 4(b) shows the threshold variation of a static particle under the influence of hydrodynamic lift forces. The particle resistance fluctuates opposite to the lift force fluctuations with a factor of $-\tan (\theta+\beta)$, which is -0.357 in this case. As shown in the marked section, negative lift forces (downward) induce the extra resistance by increasing the counter moment to prevent particle motion. A similar observation was reported by Celik et al. (2013). Note that the moment of lift forces is incorporated in the torque computation (equation (3)). Therefore, they are ruled out in the threshold determination (equation (7)) to avoid redundancy in this simulation. The threshold now appears in a function of the resistance angle, i.e. the in situ threshold.
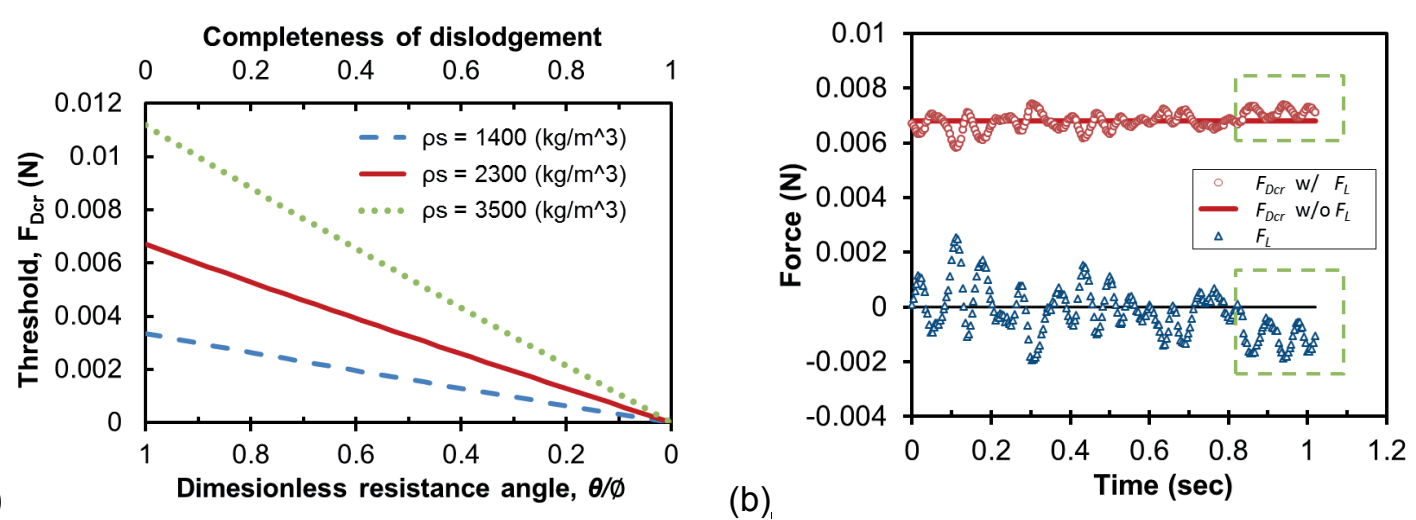

Fig. 4 (a) Dynamic particle resistance. (b) The effect of lift forces on resistance. 
Threshold variation coupled with fluctuating fluid forces complicate the prediction of dislodgement, because now the particle dynamics are nonlinear and time-dependent. For better understanding of this intriguing phenomenon, the applied fluctuating drag forces and the in situ critical value are illustrated in the following section.

\section{MECHANICAL DETAILS}

Figure 5 shows the corresponding mechanical details of the previous three migration scenarios (Fig. 3(b)-(d)). Prior to the incipient motion, a constant threshold, $F_{D c r}=0.0067$ (N), corresponds to a static sediment particle in the base pocket. Once this particle gets entrained, the threshold varies with particle trajectory, i.e. the in situ $F_{D c r}$.

Within a prompt migration (Fig. 5(a)), the in situ threshold decreases monotonically in the dislodging progress. Though the drag force diminishes before achieving dislodgement, the intensely reducing threshold secures a positive force parcel. The integration of this force parcel, i.e. impulse, converts into potential energy of the particle by raising it, and into kinematic energy of the particle by accelerating it. Note that a milder slope of the force trace than that of the threshold is a key factor of prompt dislodgement. No intersection of these two slopes is observed before full dislodgement. To achieve this, the energy transfer in the early stage needs to substantially lower the energy barrier (threshold) by relocating the particle to a less resistant position. Otherwise, a negative force parcel may appear and retard the particle motion, as in Fig. 5(b) and (c). A single positive parcel containing sufficient energy is the major feature of prompt dislodgement.

In Fig. 5(b), two positive force parcels plus a negative one result in a tardy dislodgement. Apparently, the first parcel lowers the threshold but the reducing rate does not compare with that of drag forces. Once the dropping force trace intersects the threshold, an adverse torque due to gravity is exerted on the particle to counter its movement. Not until the subsequent positive parcel, the sediment particle decelerates to zero velocity and even reverses to the resting pocket. Figure 3(c) shows this reverse trajectory in the section of reducing completeness. By comparing their commencement, a time lag between the negative parcel and reverse movement is noticed. The lag is the duration to decelerate the advancing particle to the reverse point, and the span of that lag is determined by the existing particle momentum gained from the first parcel.

The second positive parcel in Fig. 5(b) accelerates the particle again before it falls on the pocket bottom. Due to the reduction in resistance, the second accelerating stage succeeds in fully dislodging the particle, even though the magnitude of forces in this stage is smaller than the precedent stage. Given the peak force deviates from the dislodgement, the magnitude of forces is not suitable to predict the dislodgement point. More important, the single-parcel approach is not suitable for such multi-parcel scenarios. As shown here, the required energy can be accumulated over several parcels, and hence, a continuous multi-parcel approach is necessary to improve the prediction.

Similarly, Fig. 5(c) shows a multi-parcel case but with a higher frequency in the force fluctuation. With short duration, each positive parcel contains deficient energy yet the combination of the four parcels suffices to dislodge the sediment particle. The first parcel commences the movement but ends up in vibration around the resting location. Nevertheless, the energy contributed by this parcel is fractionally preserved in the vibrating sediment particle through the interchangeable potential and kinematic energy. Analogous to a sphere rolling back and forth on a curved plane, the potential energy of the raised particle partially remains during the vibration. This preserved energy, however, may not be represented with the reduction in the threshold. Due to the in situ threshold merely accounts for potential energy (particle trajectory), the energy that purely appears as the kinematic sort would be neglected when the particle falls on the bottom with momentum. The preserved energy combined with the subsequent three parcels leads to the dislodgement 0.3 seconds after the incipient motion.

Dislodgement of sediment particles may result from several positive drag force parcels; each of them is inadequate to dislodge the particle by their own energy. More precisely, a particle is intermittently stricken by individual force parcels and would be advanced further at a time as far as 
(a) Drag over prompt dislodgement

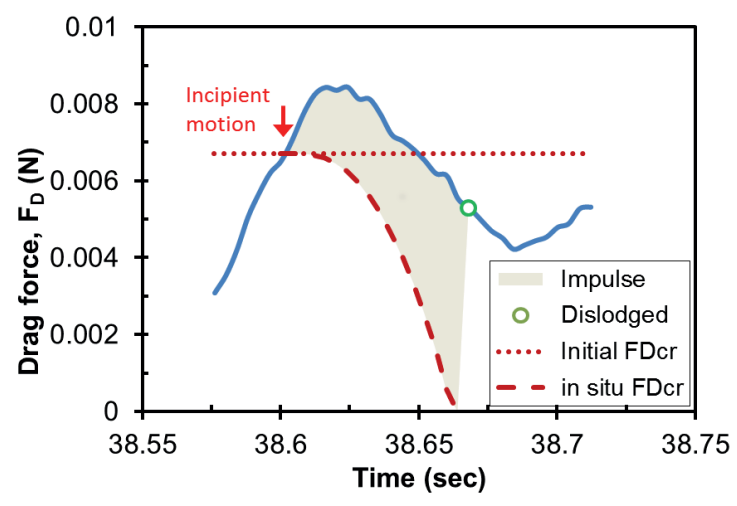

(c) Drag over vibrational tardy dislodgement

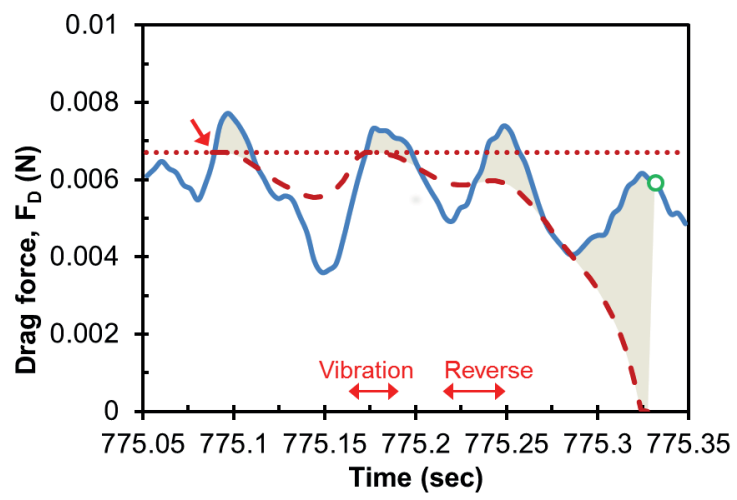

(b) Drag over tardy dislodgement

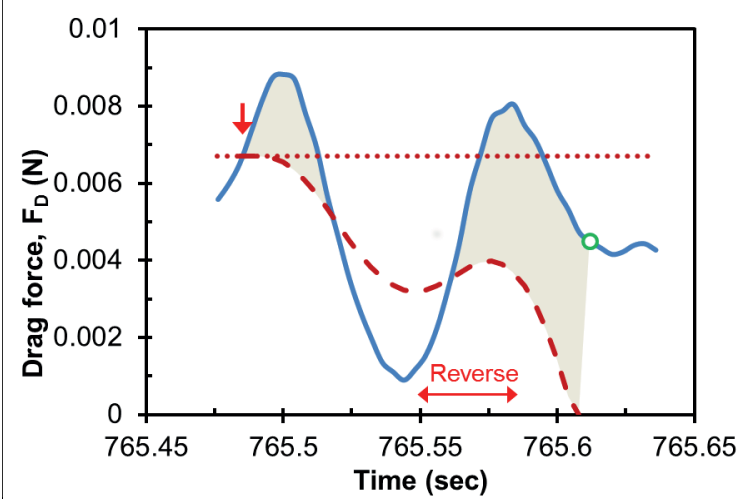

(d) Above-threshold dislodgement

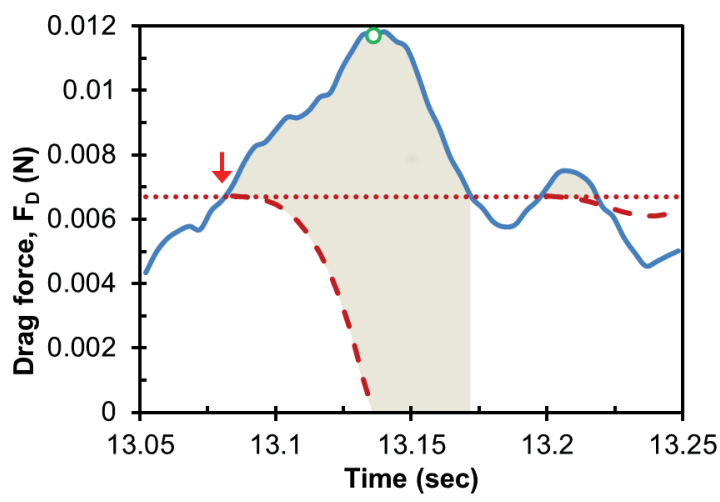

Fig. 5 Types of dislodgement.

that parcel can carry. This scenario emphasizes the necessity of a continuous model to depict the energy accumulation on a particle.

The present model also sheds light on turbulent structures instead of instantaneous fluctuations in terms of dislodging a particle. Given the above multi-parcel cases, particle migration requires energetic flow structures that span sufficient duration to impart necessary momentum. Some studies have attributed dislodgement to the retrograde vortices in turbulence (Dwivedi et al., 2011; Wu \& Shih, 2012), but the scale of those vortices remains undetermined. In Valyrakis et al.'s report (2013), the longitudinal length of energetic structures ranges extensively from 0.4 to 8 times the grain size. Nevertheless, a minimal scale of twice the particle diameter is recommended by them to secure adequate flow energy. This model, instead, suggests 0.9 to 1.7 times the particle size.

In Fig. 5(a)-(c), different turbulent structures are noticed through the frequency of fluctuating drag force. The frequency of drag fluctuation increases from about $4 \mathrm{~Hz}$ in prompt migration (Fig. 5(a)) to $11 \mathrm{~Hz}$ in vibrational tardy dislodgement (Fig. 5(c)). Provided one frequency corresponds to one particular turbulent structure: as known, low frequency to the energetic structure; high frequency to the feeble structure, this model reveals a relationship between the migration type and the flow structure. For prompt motion, low frequency (long duration) energetic structures assure adequate energy in the early dislodging stage for the monotonic advance. On the contrary, high frequency (short duration) feeble structures usually lead to tardy migration with/without vibration.

As well-known, a turbulent flow has a wide range of fluctuation frequencies; hence, particle dislodgements presumably appear in various scenarios. Due to their own limitations, different prediction models may be biased toward/against particular migration types. In the next section, therefore, we elaborate on the uncertainty of commonly used models. 


\section{UNCERTAINTY OF DISLODGEMENT PREDICTION}

The possible inaccuracy in dislodgement prediction is elaborated for three models, namely the force magnitude-based model, single-parcel impulse model, and multi-parcel impulse model. For convenience, hereafter they are referred to as the magnitude model, single-parcel model, and multi-parcel model, respectively.

In a magnitude model, particle dislodgement presumably occurs at (or close to) the peak drag force above the threshold (Fig. 5(d)). For clarity, the "threshold" here is referred to the initial threshold instead of the in situ one. This paper, however, sheds light on the possibility of the under-threshold dislodgement (Fig. 5(a)-(c)). This sort of movement is readily omitted by the magnitude model because of its lack of temporal aspects to account for energy accumulation and the reducing in situ resistance. The present simulation shows that the ratio of above-threshold migrations to under-threshold ones approximates to 1:1. In other words, this magnitude model may underrate the migration by a factor of 0.5 . However, this model was also reported overrating the dislodgement by ten-fold if it mistakes the force peaks as dislodgements (Diplas et al., 2008). In short, the magnitude model tends to collectively overvalue the migration, though the underthreshold ones are overlooked.

By incorporating force duration, a single-parcel model significantly improve the prediction. However, as shown previously, this discrete approach is biased against the multi-parcel movement and the under-threshold migration (Fig. 5(a)-(c)). An underestimate may occur in this model.

The multi-parcel model proves to be a suitable approach to depict particle dynamics. It upgrades the single-parcel model with the real-time particle response, which alters the threshold of particle motion. Both above-threshold and under-threshold dislodgements are detectable with this model, i.e. no underestimation exists, and the overestimation that compromises the magnitude model is also rectified by incorporating impulse parameter.

\section{CONCLUSIONS}

The purpose of this paper is to provide evidence to support the advantage of the multi-parcel impulse approach to predict particle dislodgement. Here we employ a theoretical model to simulate particle dynamics under the influence of hydrodynamic forces. For simplicity, a perfect rolling mode is assumed for particle motion.

Three movement scenarios are found in the simulation: prompt dislodgement, tardy dislodgement and vibrational tardy dislodgement. Each scenario corresponds to a particular flow pattern and mechanical aspects. In addition, a dynamic threshold is presented as a function of particle trajectory.

The preference of different models for types of inaccuracy is indicated: the magnitude model tends to overvalue the dislodgement, whereas the single-parcel model tends to undervalue it. The multi-parcel model, in all, is the most accurate approach without over- or underestimation.

Acknowledgement The support of the National Science Foundation (grant CBET-1033196) for this study is gratefully acknowledged.

\section{REFERENCES}

Celik, A.O., et al. (2010), Impulse and particle dislodgement under turbulent flow conditions. Physics of Fluids (22), 046601, doi: $10.1063 / 1.3385433$.

Celik, A. O., et al. (2013), Instantaneous turbulent forces and impulse on a rough bed: Implications for initiation of bed material movement. Water Resources Research 49(4), 2213-2227.

Diplas, P., et al. (2008) The role of impulse on the initiation of particle movement under turbulent flow conditions. Science 322(5902), 717-720.

Dwivedi, A. et al. (2011) Flow structures and hydrodynamic force during sediment entrainment. Water Resour. Res. (47), W01509, doi:10.1029/2010WR009089.

Julien, P. Y. (2010) Erosion and Sedimentation. Cambridge University Press. 145 - 147.

Lee, H., Ha, M. Y. and Balachandar, S (2012), Work-based criterion for particle motion and implication for turbulent bed-load transport. Physics of Fluids 24(11), 116604.

Valyrakis, M., Diplas, P. and Dancey, C. L. (2013), Entrainment of coarse particles in turbulent flows: An energy approach, Journal of Geophysical Research: Earth Surface 118(1), 42-53.

Wu, F.-C., and Shih, W.-R. (2012) Entrainment of sediment particles by retrograde vortices: Test of hypothesis using nearparticle observations, J. Geophys. Res.(117): F03018, doi:10.1029/2011JF002242. 